\title{
Integration of GNSS-IMU for increasing the observation accuracy in Condensed Areas (Infrastructure and Forest Canopies)
}

\author{
Mokhamad Nur Cahyadi ${ }^{1}$ and Irene Rwabudandi ${ }^{1}$ \\ ${ }^{1}$ Geomatics Engineering Department, Institut Teknologi Sepuluh Nopember (ITS), Surabaya, Indonesia
}

\begin{abstract}
Position determination using satellite navigation system has grown significantly. It provides geospatial with global coverage called GNSS (Global Navigation System Satellite). GNSS satellites consists of GLONASS, GPS (Global positioning system) and Galileo.GPS is the most commonly used system and it is known to its capability to determine 3D position on the surface of the earth. In order to determine the position, a GPS receiver must be able to receive signals from at least four GPS satellites. However, the determination of position in condensed areas such as tunnels, area surrounded by high rise buildings, highly forested and in other closely-knit areas is not achieved because satellite signals cannot reach the receiver in the abovementioned areas and also others where the signals are reflected before being received by a GPS receiver. In this paper, we present the algorithm to fuse GPS and the inertial measurement unit (IMU) to enable positioning in the above-mentioned Condensed Areas. The standard deviations of the two measurements show that GPSIMU is better than GPS alone, the standard deviation when satellite outages occurred is -4.57475 for GPSIMU measurements and 0.218675 for GPS observations. We presented the results in graphics and it shows that GPS measurements are easily disturbed by external influence such as multipath but GPS-IMU graphic is continuous and robust. The advantages and disadvantages of GPS and INS are complementary and make them work together to enable the accurate measurements in the areas mentioned above. Integration of INS and GNSS can be classified into three types, loosely coupled Kalman filter, tightly coupled Kalman filters and ultra- tight coupled Kalman filter. In this research we used loosely coupled Kalman filter and tightly coupled Kalman filters to combine GPS and INS in one system.
\end{abstract}

\section{Introduction}

Nowadays, researchers have been recognized the advantages of combining the Global Navigation satellite system with the inertial navigation sensors. GNSS measurements encounters several errors due to the space segment, the user or receiver locations, signal propagation etc. [1]. The idea behind GNSS positioning is that at least four satellites must be visible in the horizon of the receiver. However, this conditions in not fulfilled in the extended GNSS signals denied environments such as in the tunnels, urban area with high rise buildings, in very dense forests and in other indoor environments. A further development is the INS (Inertial Navigation System) sensor application with acquisition data rate about 200 $\mathrm{Hz}$, which the capability is greater than the GNSS data rate. The integration of GNSS and INS, has advantages in accuracy of measurement, especially in areas where satellite signals cannot be received by receiver.

Inertial Navigation System is defined as the sensor which represents sensitivity in the force and rotation. The force in this case is due to gravitational field of the earth, whereas the rotation in this case is due to rotation of the earth. This system consists of three main parts; the first part is gyroscope as the rotation sensor, the second part is accelerometer as the motion sensor and the third part is computer which is used to calculate the movement of vehicles [2]. Gyroscope and accelerometer observe continuously in position, velocity and attitude, in which the calculation is conducted in the specified reference frame with certain formula. An inertial navigation system determines components of the position, velocity and attitude by integrating acceleration and rotation which is occurred in the inertial reference system (such as body reference frame or global reference frame). The errors which is occurred in this sensor increases linearly as function of time. In reality, there are several applications of inertial navigation system, such as aircraft navigation, submarine, ships, and the strategic of the missile.

The Integration of the INS and GNSS have a such phenomenon which the INS sensor generates the position, velocity and attitude in faster rate than GNSS sensor. The INS sensor consists of triad accelerometer (for measuring acceleration rate), triad gyroscope (for measuring rotation rate) and a computer which is used to do calculation (Munguia, 2014). The calculation means integrate the position, velocity and attitude. Then the result of this calculation tuns out to be the output of INS sensor. So, the error that occurred in this process is due to position, velocity and attitude. The error is potentially unbounded.

\footnotetext{
* Corresponding author: cahyadi@geodesy.its.ac.id
} 
On the other hand, GNSS receiver that generates position and velocity has bounded error. Actually, GNSS also can be used to observe the attitude. However, attitude measurement is more complicated, so INS sensor was chosen for attitude measurement. The output quality of the GNSS sensor has bounded error in position and velocity measurement. Moreover, it can be concluded that GNSS and INS sensor have complementary attributes. It means that position and velocity estimation from GNSS can also be used as the calibration process of the INS output (position, velocity and acceleration). The application of the INS is also important when the satellite outage of the GNSS occurs.

As described above, there are some weakness and strengths in GPS application, as well as INS application. GPS has a high accuracy in the determination of position in a long period of time. However, the GPS cannot work properly when located in areas where satellite signals cannot be received by a receiver. For example, in the areas surrounded by many high-rise buildings or measurements conducted in the tunnel. In this case, buildings cause multipath, so satellite signal is not directly received by the receiver. The signals are reflected by buildings and then received by a receiver. On the other hand, in the tunnel, receiver cannot receive satellite signals. The objective of this research is to Integrate GPS-IMU for Increasing the Observation Accuracy in Dense Areas, with respect to the error behavior of each system, and the relationship between the satellite outage and accuracy generated by each system.

\section{Measurement Systems}

The objective of this research is to combine GPS with IMU to enable positioning in the satellite's signals attenuation areas.

\subsection{Inertial navigation Unit (IMU)}

IMU is a sensitive device consists of three main parts; the first part is gyroscope as the rotation sensor, the second part is accelerometer as the motion sensor and the third part is computer which is used to calculate the movement of vehicles. Gyroscope and accelerometer observe continuously in position, velocity and attitude, in which the calculation is conducted in the specified reference frame with certain formula. A computer performs signal conditioning and temperature compensation, the accelerometer and gyroscope data outputs are velocities and angular rates via a serial interface Computer at a rate of $100 \mathrm{HZ}$ to $1000 \mathrm{HZ}$ [3].

\subsection{Inertial Navigation System (INS)}

The inertial navigation system principle stands for solving Newton's equations of the rotation of the earth, this has made possible by integrating the acceleration and angular rates sensed by IMU [3]. The following process is to initialize the inertial navigator with known position and velocity from GPS and aligned with respect to the vertical and true North. After the alignment, the inertial navigator has established a local level mathematical frame of reference called the navigation frame whose heading with respect to North in this case the orientation of IMU must be known.

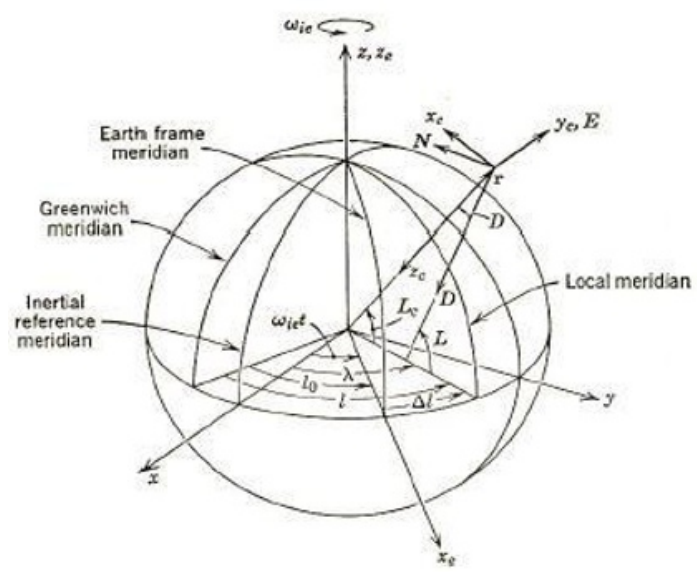

Fig. 1. Reference frames used in INS [3]

\section{Results and Analysis}

The objective of this study is to test and compare IMUGNSS and GNSS alone measurements in dense area, we also compared the RMS errors of two systems and the relationship between the satellite outage and accuracy generated by each system. The data used was collected in Tainan near National Cheng Kung University. Both methods were evaluated with different solutions, forward and smoothing operation. Data post processing of GNSS solutions will also be compared with the integration of these two methods. Reference stations used in this research are three different reference stations.

In this research, two methods were compared to each other. The first method is GNSS only. In this method, two main processes, data post processing of GNSS solution are conducted by one process only. The first step is calculation of the GNSS post processing and the second step is integration of result of GNSS post processing with INS. The second method of integration navigation system is loosely coupled Kalman filter which is solved by Geopointer software. In this software, both two main steps of the calculation, which are GNSS post processing and integration of the GNSS- INS, are solved separately.

\subsection{The Kalman filter process}

The Kalman filter process is a mechanization which takes dynamic model of the system, measurement, deterministic input with the certain algorithm to blend all of the input parameter to get estimation [4]. This section will explain the basic concept of the linear Kalman filtering.

The equation can be explained in the following way. The is an $(n x l)$ state vector, $(t i, t i-1)$ is an (nxn) system transition matrix, $G(t i-1)$ is an (nxs) noise input matrix, and $w(t i-1)$ is an $(s x l)$ white Gaussian noise (Bulut, Cavanaugh, \& Bernal, 2010):

$$
x\left(t_{i}\right)=\Phi\left(t_{i}, t_{i-1}\right) x\left(t_{i-1}\right)+G\left(t_{i-1}\right) w\left(t_{i-1}\right)
$$




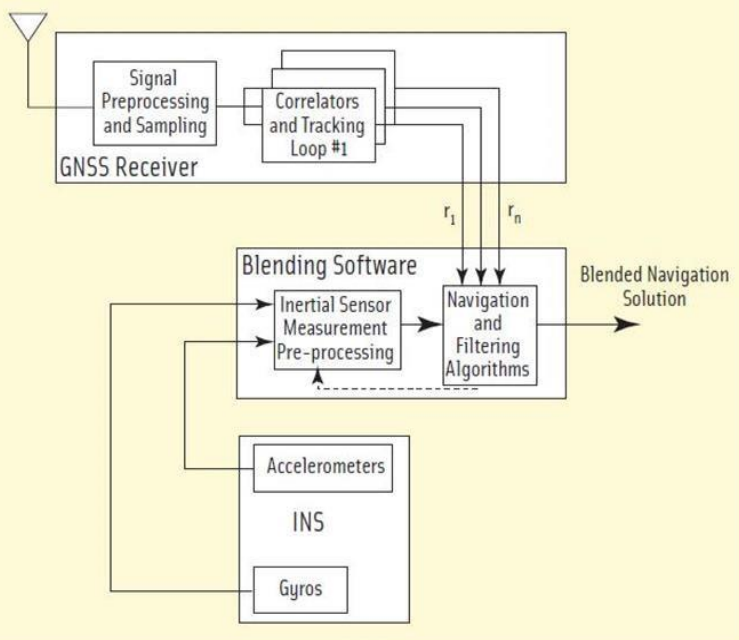

Fig. 2. The Kalman filter process

The non-linear observation is written as follows:

$$
Z\left(t_{i}\right)=h\left[x\left(t_{i}\right), \mid t_{i}\right]+v\left(t_{i}\right)
$$

Where $Z(t i)$ is an $(m x l)$ measurement vector, $h[x(t i), t i)]$ is an $(m x l)$ non-linear measurement model vector, and $v(t i)$ is an $(m x l)$ white Gaussian measurement noise vector.

Kalman filter can be divided into several sequences in three steps, initial condition information, filter propagation, and filter update.

\subsection{Kalman Filter Initial Condition Equations}

The equation in this process can be written as follow:

$$
\begin{aligned}
& \hat{X}\left(t_{0}\right)=E\left\{X\left(t_{0}\right)\right\}=\hat{X}_{0} \\
& P\left(t_{0}\right)=E\left\{\left[X_{0}\left(t_{0}\right)-\hat{X}_{0}\right]\left[X_{0}\left(t_{0}\right)-\hat{X}_{0}\right]^{T}\right\}=P_{0}
\end{aligned}
$$

This is detailed by [5].

\subsection{INS/GPS Integration}

In this research, we use Geopointer to integrate GPS with INS.GPS data were collected separately with GPS-IMU data. This software is used to process GPS data to provide time, position and velocity, to process IMU/GPS integration data in the loosely coupled and tightly coupled integration, apply the extended Kalman filter (EKF) and smoothing in data processing to provide optimal navigation solutions including position, velocity and orientation, to provide functions for coordinates transformation, to provide graphical interface to display graphical navigation solutions and overlay map and finally it provides a textual editor interface to show and edit text.

The result of this research shows the comparison between GNSS and GNSS-IMU when satellite outage occurs. It's located in the area surrounded by many buildings and trees. Hence, GNSS receiver cannot obtain satellite signals in short of time. The error occurred before satellite signal outage in this area is multipath error, because of many high-rise buildings, it could be also seen from the DOP and VDOP value is 6.7. This phenomenon can be seen from the error of the GNSS solution only increased before satellite outage occurred. The value of rms error during satellite signal outage is getting worse. This condition shows that GNSS-IMU is 25 up to 45 times better compare to GNSS solution only. GNSS solution only has a problem when there is a loss of GPS signal or phase ambiguity from cycle slip. It could be seen from the figure after satellite signal outage during 45 seconds. The value of rms error requires more or less 4 seconds to become stable. It could be understood, because in the GNSS solution only, INS data cannot be used for ambiguity estimation. On the other hand, GNSS-IMU integration has a character of faster ambiguity estimation.

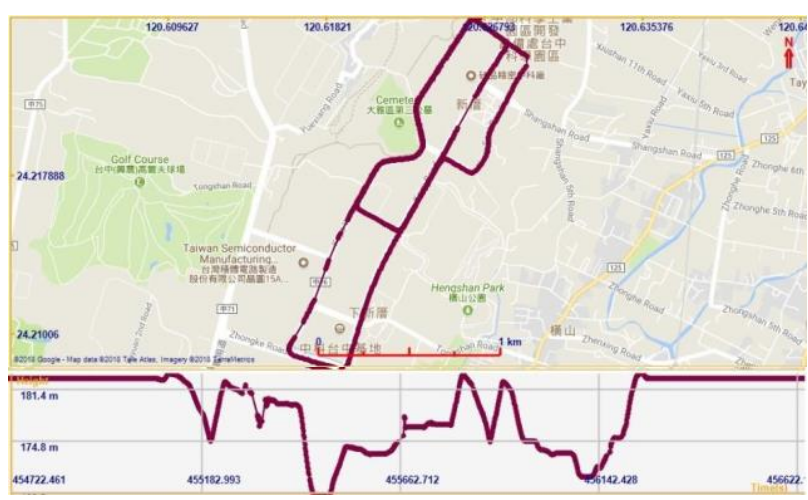

Fig. 3. GPS measurements trajectory

The figure above represents GPS measurements before being integrated with IMU, the trajectory is not continuous because the satellite outages occur several times. GPS recorded the data every single second. We computed the average time of the satellite outage and we found out that in 56 seconds GPS receiver was not recorded the data due to obstacles that surround the area.

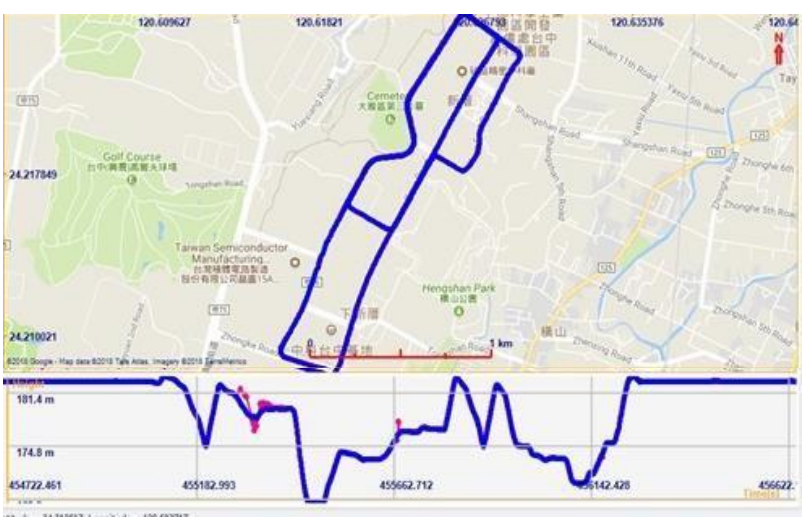

Fig. 4. GPS-IMU trajectory

The figure above shows the result of INS/GPS integration, GPS-IMU result shows that the system cannot be affected by external influences such as multipaths as shown in the figure 4 GPS-IMU makes a continuous, robust, and smooth trajectory and this made the system better than GPS measurements alone in figure 3, the trajectory is not continuous because of the occurrence of satellite outage. The standard deviations of the two measurements show that GPS-IMU is better than GPS alone, the standard 
deviation when satellite outages occurred is -4.57475 for GPS-IMU measurements and 0.218675 for GPS observations.

\section{Conclusion}

GNSS measurements encounter various errors when observing in an extended signal denied environment. The satellite outages affect the observation accuracy. However, INS/GPS integration is result in a very strong system that cannot be easily disturbed by satellite signals attenuation. In this paper we conclude that GPS-IMU system is far better than GPS system alone, this has proven by the comparison of RMS error of the two system. The RMS error of GPS system bigger than the RMS error of INS/GPS system and the trajectory graphics of the two systems show clearly that INS/GPS system is stronger than GPS alone.

\section{References}

1. Seeber, G. Satellite Geodesy. New York: Walter de Gruyter. (2003).

2. Munguia, R. A GPS-aided Inertial Navigation System in Direct Configuration. Journal of Applied Reasearch and Technology, 803-814. (2014).

3. Zhou, J., \& Bolandhemmat, H. Integrated INS/GPS Syatem for an Autonomous Mobile Vehicle.

4. Bulut, Y., Cavanaugh, V. D., \& Bernal, D. Process and Measurement Noise Estimation for Kal,an Filtering. Proceeding of the IMAC-XXVIII. (2010).

5. Suzdaleva, E. Initial Condition for Kalman filtering: prior knowledge specification. Proceeding of the 7th conference on 7th WSEAS International conference on systems Theory and scientific Computations, 4549. (2007) 